\title{
The teacher as a mediator in a networked society
}

\author{
Andrée Klein and Hélène Godinet \\ Institut Universitaire de Formation des Maîtres, 30 avenue M. Berthelot, 38100, Grenoble, \\ France.andrée.klein@grenoble.iufm.fr
}

Keywords: teacher's role, multimediated learning, networking, language learning, mediation

\begin{abstract}
As an increasing number of schools are being connected to the Internet and equipped with telematics tools, there is a growing fear among teachers who do not know how to make effective use of the machines. In this paper, we are concerned to pinpoint through our day to day practice as teacher trainers, and our involvement in European projects, the competencies that emerged from our activities with our students. The paper defines the essential role of the teacher regarding ICT, examines the changing role of the teacher in an ICTbased school environment and a networked society, and calls for vigilance in the use and integration of ICT in education and teacher training.
\end{abstract}

\section{INTRODUCTION}

Today, there is a widespread belief that, with the advent of ICT, networks and hypermedia, the role of the teachers will change, and that the teacher will no longer be the only one to convey knowledge. These glib and typically superficial ideas provoke as many fears as enthusiastic reactions among the teaching profession. Some fear that computerised teaching gradually take over or distance learning will supersede face-to-face teaching. Others are enthusiastic because they support the notion of a radically new and improved educational paradigm and wish to infuse into the profession an innovative spirit.

It is clear that when new technologies are incorporated, they are no doubt important when they serve to overcome material and technical obstacles to teaching and learning. But there is no clear evidence, on a larger scale, that ICT, networks and hypermedia in themselves are sufficient as tools aimed at

The original version of this chapter was revised: The copyright line was incorrect. This has been corrected. The Erratum to this chapter is available at DOI: 10.1007/978-0-387-35499-6_29 
adding value to the educational process, or at improving the quality of education and its output. Nevertheless, from our day to day practice as teacher trainers, we have a body of experience and now have reasons to believe that this particular combination of pedagogies and technologies is likely not to revolutionise educational practice, but rather to bring about some noticeable changes, especially in the role of the teachers.

Firstly, we will present these changes in terms of the competencies needed to grapple with ICT and the roles that must be held by teachers. Secondly, we will try to re-position the use of ICT and reflect on it in a more general context, to gain a better understanding of where we stand and assess the credibility of some routes in initial teacher training.

\section{DEFINING A PROGRESSIVE EVOLUTION IN TEACHING COMPETENCES}

Starting from our specific framework of language learning and teaching, we have been experimenting for some years with our student-teachers some practical ways of integrating ICT. We believe that through a set of key functionalities of interactivity, multimodality, and evolutivity, hypermedia have contributed to that ICT integration is possible and can transform the teaching and learning environment.

Over the years, we have tested different software and CD-ROMs, organised online tutoring via email and video-conferencing, participated in online virtual workshops, shared good practice via video-conferences, used and designed online resources. We have worked with European partners, in Socrates projects like APPLAUD and T3 (telematics for teacher training; a telematics application programme for education and training supported by the European Commission DG XIII). From these various observations and experiments we have identified three types of competencies to be developed in teacher training. They are technical, pedagogical and communicative abilities.

\subsection{Technical ability}

Competent teachers can use a wide range of appropriate telematic resources, such as browsing and/or creating networked resources, email, video-conferencing, to enhance personal and professional efficiency, and to update skills in the light of new developments. 


\subsection{Pedagogical ability}

Competent teachers can plan and implement lessons with telematics taking into account the needs, experiences and abilities of individual students. They can organise resources effectively, to ensure differentiation and progression, monitor and evaluate the progress of students and the use of telematics and the outcomes. They can manage the classroom appropriately according to their teaching objectives when using telematics.

\subsection{Communicative ability}

Competent teachers can optimise the interaction between medium and message in order to adjust to the mode of communication. To use hypermedia and networks, teachers need to understand that information and resources are open, virtual, distant, multimodal. Networking opens schools to other contexts, other communities and other cultures. Telematics tools introduce opportunities to 'pick up' non-verbal communication cues such as gesture, body language, facial expression and human behaviour in general.

Learning and teaching a language involves studying another culture and accepting the 'otherness' in it. Learning and teaching a language includes cultural and factual knowledge, e.g. of history and current events. It is not only learning texts but more increasingly understanding sound, hearing, interpreting pictures and video. And finally, teachers have to plan and deliver hypertextual rhetoric, and evaluate and assess their students' learning in dealing with non linear networked information. The competencies identified here, though not exhaustive, imply a re-organisation of the roles of the teacher.

\section{DEFINING THE CHANGING ROLES OF THE TEACHER IN A NETWORKED SOCIETY}

Reflecting on social and human artefacts and activities, we are struck by the fact that they all are the results of a mediation. For example, there is no science, no religion without mediation. Education falls into the same category and an important message to deliver is that there is no education without mediation. Therefore, the role of the teacher is above all that of a mediator i.e. the one who verbalises experience and knowledge to ensure successful acquisition.

This fundamental role has some very practical implications when it comes to integrating ICT and can be seen as a core feature to be broken up into several parts.

- The teacher as a designer;

- The teacher as an expert; 
- The teacher as a tutor;

- The teacher as an evaluator.

\subsection{The teacher as a designer}

The teacher as a designer is a mediator between the students and the environment. Authentic learning environments are ones which allow participants to gather information from multimedia data bases, and to communicate with others via multimedia technology. In term of resources, the web or ICT based packages form a universal library.

In terms of collaborative work, with teachers and students or students and experts outside the classroom, real-life communication can be established with peers in other countries, by email or video-conferencing. Project work can be shaped around sharing and negotiating. It may involve a reorganisation of pedagogical time and places, reshuffling teaching hours, and rethinking classroom management. The teacher can turn their classroom into an open, international space, thus adding to it a virtual dimension.

\subsection{The teacher as an expert}

The teacher as an expert is a mediator between the students and knowledge. The teacher's traditional task is to organise and structure information. It is even more so with ICT because they have to turn the vast collection of information, sometimes uncontrolled, into knowledge. The teacher decides whether a site meets their educational objectives; tailors the materials to suit them; helps students to discriminate between information and the processing of relevant information; and stimulates the students to use online resources in a shrewd, cautious manner to form a critical community of users.

\subsection{The teacher as a tutor}

The teacher as at tutor is a mediator between the students and their learning styles. Electronic online tutoring enables the teacher to have frequent feedback and therefore an acute idea of his students' learning and cognitive styles. Edward (1997) relying, on Anglo-Saxon sources on cognitive psychology, showed how hypermedia enable the interaction between contents, methods and learning.

This will enhance the traditional moral support given by teachers. They can tutor not only the students' products but the process of learning. The computer provides the students with a learning programme, the tutor organises the content according a progressive approach adjusted to the individual needs of the student. The student needs to receive, in real time, a 
feedback which is more than a computer-calculated result. The role of the teacher is to structure their pedagogical online material and create hyperlinks in the provided resources. In this way users get an appropriate feedback, when browsing and re-structuring information, which may, when necessary, have been pre-selected by the teachers.

\subsection{The teacher as an evaluator}

The teacher as an evaluator is a mediator between the students and their productions. The teacher provides their students with clear insights into their progression, storing files of their work, defining their weak and stronger points to help them come to terms with their learning and cognitive styles.

After engaging in these experiments that have led us identify and define competencies and roles, we must acknowledge the fact that teaching with ICT is an uphill task. The teacher has to reflect on their practice and on the learning processes and procedures even more than in a traditional learning environment. They have to become - which may be quite new with teachers - a professional.

\section{THE TEACHER IN A NETWORKED SOCIETY : A WATCHFUL MEDIATOR}

The teacher has not only to reflect on their practice but much more on the interaction between school and society. They have to reflect on the pedagogical relevance of ICT. A reflective attitude is possible only if at some point the teacher can take up a critical attitude. To keep distance from the 'wired-up' society, to ponder on the forceful hype around ICT (pros and cons alike) is the best way to escape unscathed. A cautious approach, as suggested Wolton (1997), supported by a strong sense of discrimination and relativity, must be taken to escape the 'cycle of deception' promoted by manufacturers and some researchers in order to give credible answers to the call for evidence coming from our colleagues.

Where do we, teachers and educators stand in the 'information society'? How can we find our way through the 'webby' maze and find suitable strategies to integrate ICT and train student teachers? Here again, the teacher is a mediator between what is going on in both society and school. Information and communication technologies are, so it seems, vested with an intrinsic ability to solve social and cultural problems. We actually cannot demonstrate that a rich authentic ICT-based learning environment will solve them. If the mediation is effective in the classroom and at school, it also has to be effective in society. Actually, about ICT, the teacher has to be able to distinguish between different levels and services. 


\subsection{Between different levels}

The three fields, that is of innovation (discovery), of application (industrial implications) and of service (usage) overlap, and there is nothing worse than confusing them. Economists and manufacturers are inflicting the effects of the raging battle waged to occupy the unchartered lands of the ICT market on us. The consequences are that they tend to impose their rhythm, their pace of change, and their vision of the future, shaping it with the improbable concept of information society. We must clearly state that we are playing in a different field.

\subsection{Between services}

The use of ICT in services varies considerably. While they may be useful in entertainment and tourism, it is an altogether different story with education, health and work. Oversimplification and generalisation are to be avoided in these matters. Transferability from one to the others is not that easy! The more sophisticated the activities, such as learning and teaching, the more difficult it is to standardise them.

The main question is what is access to information for? There is no relation between the increasing volume of information available and its effective use. The greater the flow of information, the greater becomes the need to acquire conceptual tools to decipher complex systems. Cultural inequalities to access and use of information will grow when going from services like tourism, entertainment to those related to teleworking, telecommuting, telematics in education. In other words, it is necessary to discriminate between the widespread availability of data and the competencies needed to process, use, and integrate them.

We have to demystify common and superficial assertions related to two notions often associated with ICT : time and autonomy. The technological literature abounds in terms of 'real time', 'speed', 'faster', 'instant access' as if all of a sudden time like space was abolished and turned into instantaneous data. Compressed time like space seems to be the new panacea that will save the world of education. As teacher trainers we have to defend and even reintroduce the term 'learning time', and state clearly that the unavoidable ingredient in the learning process is time.

Most of the time, in papers or speeches, educational multimedia software and autonomy (self-training) are synonymous. Since this new form of packaging changes the how, when and where of the teaching/learning approach, it tends to be presented as an incredible opportunity to move away from the traditional constraining learning environment, as something deceptively simple, almost magical, the ultimate in terms of individualised learning. This inordinate trend to see the individual as the nucleus of 
everything tends to deprive us of what is the most needed in our western societies, i.e. the social link or social context of learning. The social fabric is the locus of social interactions at school.

\section{CONCLUSION}

We wanted to stress the necessity to train our students not only to become capable and critical ICT users, but also and above all to become aware of the underlying concepts and the social and economic implications attached to it. We are in a transitory period, in which schools rely on tested tools and methodology. Talking about the ICT revolution does not solve most of the difficulties our societies encounter, it simply 'technisices' them. Our role as teacher trainers is to make future teachers' competencies evolve towards a progressive integration of ICT in a networked society, if such a thing exists or will ever exist. If ICT instrumentalises the mediation, it neither changes its nature nor questions its necessity.

\section{REFERENCES}

Authier, M. and Serres, M. (sous la direction de.) (1998) Le monde de l'education hors série: Apprendre à distance.

Davis, N. and Teartle, P. (1998) A core curriculum for telematics in teacher training. In G.

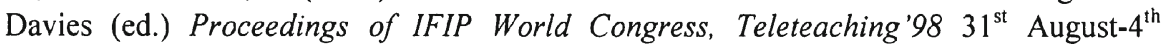
September 1998.

Dieuzeide, H. (1994) Les nouvelles technologies, outils d'enseignement. Paris: Nathan .

Durpaire, J. L. (1997) Internet à l'école en france: Guide d'usages pédagogiques. Collection l'ingénierie educative. Poitiers: Centre Regional de Documentation Pedagogique for CNDP.

Edward, N. (1997) Development of a cost effective computer assisted learning package to facilitate conceptual understanding. In Proceedings of Cal 97, April, Exeter, UK.

Linard, M. (1998) La nécessaire médiation humaine. Les cahiers pédagogiques: A l'heure d'internet. 362.

Tardif, J. (1998) Intégrer les nti. Quel cadre pédagogique? Paris: Edition Scientifique Francaise.

Wolton, D. (1997) Penser la communication. Paris: Flammarion

\section{BIOGRAPHIES}

Andrée Klein, teacher trainer at the Institut Universitaire de Formation des Maîtres, teaches English as a foreign language, foreign language methodology and pedagogy. She is an expert on integrating ICT, especially multimedia and web resources, in foreign language learning and teaching for secondary student-teachers. She is involved in different European ICT projects, and co-responsible of the T3 project. 
Hélène Godinet, teacher trainer at the Institut Universitaire de Formation des Maîtres, teaches sciences of language, integrating CAL and ICT in writing and reading. She is an expert on networking and hypertext producing and has published different papers on this subject in educational journals. She is involved in different European ICT projects, and co-responsible of the T3 project. 